\title{
Compact microscope-based optical tweezers system for molecular manipulation
}

\author{
Andy Sischka, ${ }^{\text {a) }}$ Rainer Eckel, Katja Toensing, Robert Ros, and Dario Anselmetti \\ Experimental Biophysics and Applied Nanosciences, Faculty of Physics, Bielefeld University, \\ 33615 Bielefeld, Germany
}

(Received 18 February 2003; accepted 14 August 2003)

\begin{abstract}
A compact single beam optical tweezers system for force measurements and manipulation of individual double-stranded deoxyribonucleic acid (DNA) molecules was integrated into a commercial inverted optical microscope. A maximal force of $150 \mathrm{pN}$ combined with a force sensitivity of less than $0.5 \mathrm{pN}$ allows measurements of elastic properties of single molecules which complements and overlaps the force regime accessible with atomic force microscopy (AFM). The manipulation and measurement performance of this system was tested with individual $\lambda$-DNA molecules and renders new aspects of dynamic forces phenomena with higher precision in contrast to AFM studies. An integrated liquid handling system with a fluid cell allows investigation of the force response of individual DNA molecules in the presence of DNA binding agents. Comparison of YOYO-1-, ethidium bromide intercalated DNA, and distamycin-A complexed DNA revealed accurate and reproducible differences in the force response to an external load. This opens the possibility to use it as a single molecule biosensor to investigate DNA binding agents and even to identify molecular binding mechanisms.
\end{abstract}

\section{INTRODUCTION}

The invention of optical tweezers by Ashkin ${ }^{1,2}$ pioneered the application of noninvasive micromanipulation of dielectric particles in the Rayleigh and Mie size regimes. ${ }^{3-5}$ Static and dynamic forces can be detected by using a trapped particle deflected by an external force. ${ }^{6}$ These trapping techniques have been used for manipulation of living cells, organelles, bacteria, and viruses in biology. ${ }^{7-9}$

The manipulation and control of individual molecules with optical tweezers was introduced by Block and co-workers ${ }^{10}$ with kinesin molecules, whereas Bustamante and co-workers ${ }^{11}$ investigated single- and double-stranded deoxyribonucleic acid DNA (dsDNA) molecules via attaching biotin-streptavidin functionalized microspheres to this molecules. They were able to measure the elastic response and mechanical properties of individual DNA molecules. Thus, DNA intercalating- and minor groove binding molecules and structural transitions in those molecules have also been investigated in experiments with atomic force microscopy (AFM), ${ }^{12-14}$ dual beam optical tweezers ${ }^{15}$ and micropipettes. ${ }^{16}$ Recently, it has been shown that different binding modes of DNA binders could be identified which allowed deeper insights into the molecular interplay between DNA and small ligands. ${ }^{15,17}$

In this article, we introduce a compact and versatile single-beam optical tweezers setup (see Fig. 1) which is based on a commercial inverted optical microscope. This allows high precision force measurements in single-molecule

\footnotetext{
a) Author to whom correspondence should be addressed at: Universitaet Bielefeld, Fakultaet Physik, Universitaetsstrasse 25, 33615 Bielefeld, Germany; electronic mail: andy.sischka@physik.uni-bielefeld.de
}

force experiments up to $150 \mathrm{pN}$ and ideally overlaps the force range but outclass the force sensitivity of AFM systems. ${ }^{18}$ After calibration, the setup was tested in experiments with DNA and different DNA binding ligands, showing distinct differences in the force response which allow discrimination between different binding modes.

\section{EXPERIMENTAL SETUP}

The optical tweezers system is installed on an optical tabletop (VH-3660W-OPT, Newport, CA) for vibration isolation. A diode-pumped Nd:YAG-laser (LCS-DTL-322, Laser 2000, Germany) emits at $\lambda=1064 \mathrm{~nm}$ linear polarized and collimated light $\left(\mathrm{TEM}_{00}\right)$ with a maximum power of $1000 \mathrm{~mW}$ and a beam width of $1.5 \mathrm{~mm}$. In order to reduce vibrational noise from the force signal, the original cooling fan was replaced by a solid copper heat sink and special emphasis was put to a compact and stable setup. A long pass filter (RG-850, Linos, Germany; 0.2\% transmission at 810 $\mathrm{nm}, 98 \%$ transmission at $1064 \mathrm{~nm}$ ) blocks the exication radiation $(810 \mathrm{~nm})$ of the laser pumping unit. The laser beam is redirected by two flat mirrors (10D20-ER.2, Newport, CA) into a $10 \times$ beam expander (S6A SS 0107, Sill Optics, Germany) mounted on the epifluorescence port of a commercial optical inverted microscope (Axiovert 100, Carl Zeiss, Germany). An enclosure around the lightpath prevents distortions from airflow. The laser beam passes a dichroic mirror (TFP1064nm56 ${ }^{\circ}$, Laseroptik, Germany; approximately $80 \%$ transmission at $400-700 \mathrm{~nm},>96 \%$ reflection at $1064 \mathrm{~nm}$ ) and is fed into a $60 \times$ water immersion objective (UPLAPO60W/IR, Olympus, Japan). The objective has a back side aperture diameter of $8.5 \mathrm{~mm}$ with a numerical aperture of 1.20 and a working distance of $300 \mu \mathrm{m}$. Whenever a 


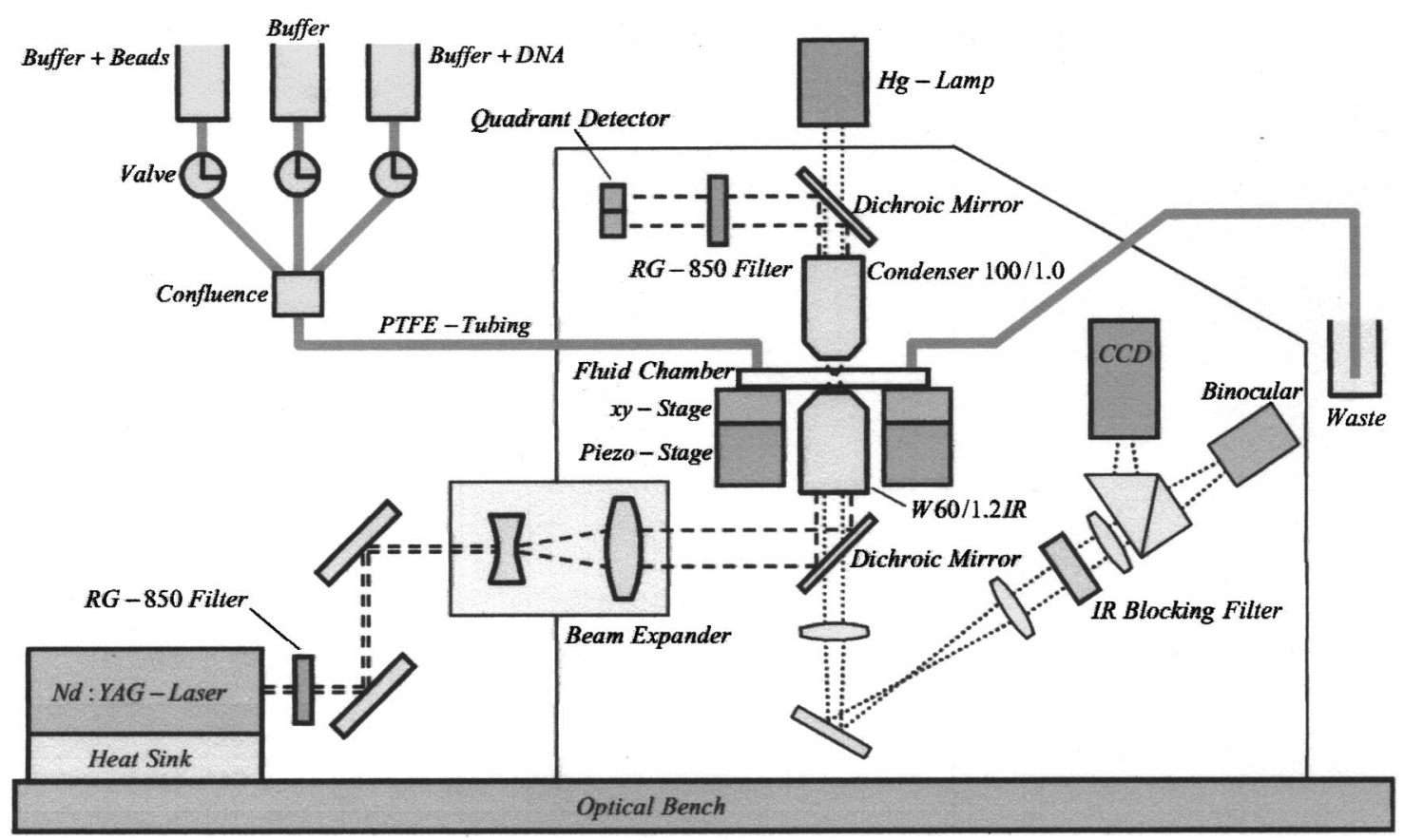

FIG. 1. Optical tweezers setup with integrated flow system.

transparent particle is trapped within the focus, the forward scattered light can be used for displacement detection. ${ }^{19}$ In our setup, the forward scattered light is collected by a water immersion condensor objective (Achroplan 100/1.0 W, Carl Zeiss, Germany). This high numerical aperture is needed (a) to collect as much forward scattered light as possible to obtain a high signal-to-noise ratio for further signal processing and (b) to observe all intensity distributions and variations inside the forward scattered beam profile. The light collected by the condensor is reflected by a dichroic mirror (TFP1064nm56 ${ }^{\circ}$, Laseroptik, Germany), passes a long pass filter (RG-850, Linos, Germany) and is position analyzed by a quadrant photodiode (SD 380-23-21-051, Silicon Detector Corp., USA) adjustable in the $x$ and $y$ directions. The quadrant detector readout (four current-voltage amplifiers, OPA111, Burn-Brown, USA) and an analog processing unit (University of Basel, Switzerland) transform the light intensity variations into a direct or normalized voltage signal proportional to the bead position in the optical trap. This voltage signal is digitized with a transfer rate of 388000 samples/s and further processed by a custom-made software based on a commercial software library (LABVIEW 6.0, National Instruments, TX). This high acquisition rate allows statistical noise averaging up to a factor of 300 per data point.

Real-time observation of the trapped particle is possible by a charge coupled device camera on the binocular side port and a mechanically detached halogen lamp. For eye and camera protection, two IR blocking filter (KG-5, Schott, Germany; $10^{-5} \%$ transmission at $1064 \mathrm{~nm}$ for each filter) are placed into the optical path.

The position control of the trap is realized by moving a fluid cell with respect to the trap via a three-dimensional piezostage (P-517.3CD, Physik Instrumente, Germany; $x, y$ : $100 \mu \mathrm{m}$ and $z: 20 \mu \mathrm{m})$ controlled by digital stage electronics.
An additional manual coarse positioning stage allows further coarse adjustments of the fluid cell.

The fluid cell is a homebuilt sandwich construction of two glass coverslips (see Fig. 2) $(60 \mathrm{~mm} \times 24 \mathrm{~mm}$ $\times 0.15 \mathrm{~mm}$ ) separated by two parafilms (American National Can, CT) where the flow channel $(42 \mathrm{~mm} \times 1 \mathrm{~mm})$ is embossed into. Two holes (1 $\mathrm{mm}$ diameter) within the upper coverslip allow liquid in and outlet. An integrated micropipette (KG-33, Garner Glass, CA) older diameter (o.d.) $=150 \mu \mathrm{m}$, inner diameter (i.d.) $=80 \mu \mathrm{m}, L=100 \mathrm{~mm}$ ) pointing directly along the flow channel was pulled (Pipette Puller P-2000, Sutter Instrument Co., CA) to form a tip with a diameter of $1.5 \mu \mathrm{m}$ with a wall thickness of less than 0.5 $\mu \mathrm{m}$. This micropipette allows mechanical trapping of individual micron-sized beads by applying low pressure.

A compact, unsusceptible, and simple liquid handling system established with teflon tubings (Bohlender, Germany; o.d. $=1.6 \mathrm{~mm}$ i.d. $=0.3 \mathrm{~mm}$ ) and driven by hydrostatic pres-

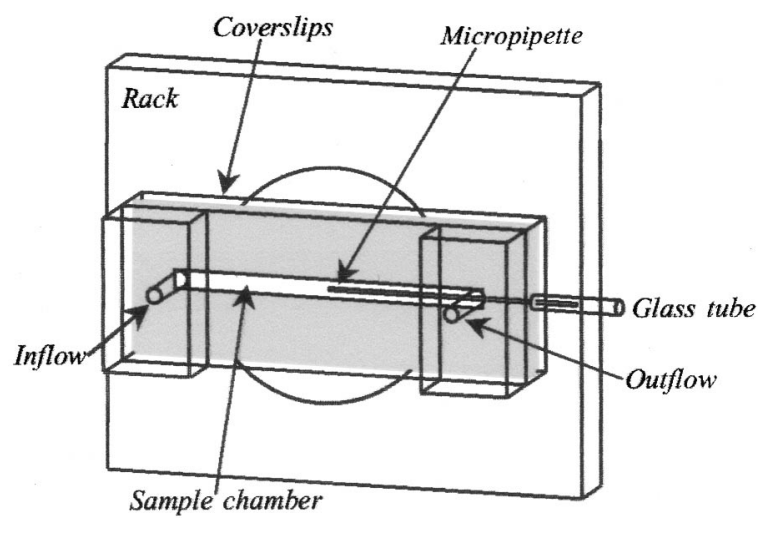

FIG. 2. The fluid chamber. 

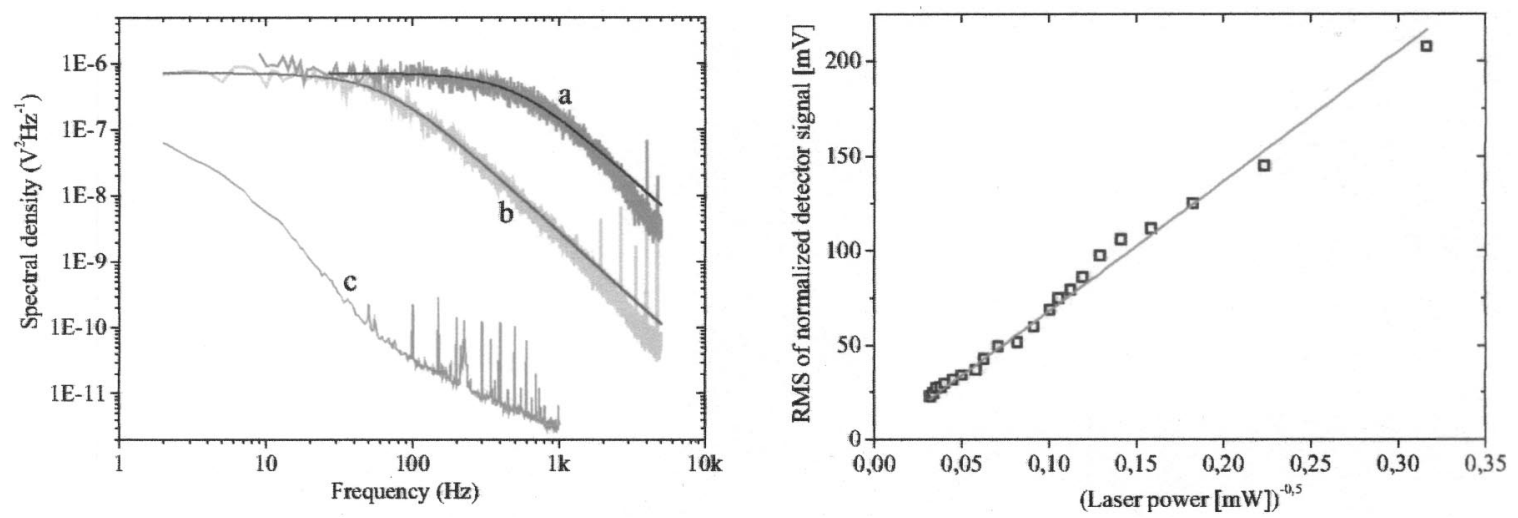

FIG. 3. (Left-hand side) Thermal noise spectra for $3180 \mathrm{~nm}$ bead (a) trapped at a laser power of $900 \mathrm{~mW}$ (corner frequency $f_{c}=502.9 \mathrm{~Hz}$, force constant $k=94.7 \mathrm{pN} / \mu \mathrm{m}$ ), (b) trapped at a laser power of $60 \mathrm{~mW}$ (corner frequency $f_{c}=63.1 \mathrm{~Hz}$, force constant $k=11.9 \mathrm{pN} / \mu \mathrm{m}$ ), (c) empty trap at a laser power of $60 \mathrm{~mW}$. (Right-hand side) Linear dependency between rms value of the normalized detector signal (proportional to the displacement of the bead inside the trap) and the inverse square root of the laser power.

sures allows reproducible, constant, and smooth liquid flow through the cell.

\section{CALIBRATION AND EXPERIMENTAL PROCEDURE}

\section{A. Trap stiffness calibration}

The stiffness of the trap was determined by measuring the maximum trapping force at different laser powers. ${ }^{8} \mathrm{We}$ used polystyrene beads with a diameter of $3180 \mathrm{~nm}$ (Spherotech, IL) covalently coated with streptavidin (binding capacity of $60 \mathrm{pmol}$ biotin $/ \mathrm{mg}$ particle). For all experiments, the stock solution $(0.5 \% \mathrm{v} / \mathrm{w})$ was diluted $1: 1000$ with $150 \mathrm{mM}$ $\mathrm{NaCl}$ and $10 \mathrm{mM}$ Tris/HCl buffer $(p \mathrm{H} 8.0)$.

The trapping force $F$ was calculated via Stokes' friction ${ }^{8}$ $F=-\gamma v(\gamma=6 \pi \eta r)$ with velocities up to $v=12000 \mathrm{~nm} / \mathrm{s}$ for a sphere with radius $r$ inside an infinite expanded homogenous fluid with a viscosity $\eta$. Trapping forces measured at $60 \mathrm{~mW}, 600 \mathrm{~mW}$, and $900 \mathrm{~mW}$ laser power were $16 \mathrm{pN}, 105$ $\mathrm{pN}$, and $145 \mathrm{pN}$, respectively. Due to losses in the optical path length we determined a final yield of $20.3 \%$ within the optical trap with respect to the laser output. Calibration of the optical trap was realized by moving the liquid cell with respect to the trapped bead at constant velocity where the voltage signal of the quadrant detector can be compared with the actual Stoke's frictional force. ${ }^{6}$

In addition, calibration was checked by computing the noise power spectrum of the detector signal by using a Lorentzian fit. The corner frequency $f_{c}$ (Fig. 3 left-hand side can be related to the force constant $k=2 \pi \gamma f_{c}$ of the optical trap potential.

A working distance of $150 \mu \mathrm{m}$ between bead and cell wall surface made corrections for drag coefficient calculations unnecessary. ${ }^{8}$ Additionally, the validity of the equipartition theorem can be reviewed: The detector voltage signal depends linearly on the displacement $x$ of the bead inside the trap. There is further a linear dependency of the force constant $k$ on the laser power $P$. We observed a linear relation between the root-mean-square (rms) value of the displacement and the inverse square root of the laser power according to the equipartition theorem (Fig. 3 right-hand side): $\langle x\rangle=\left(k_{B} T / k\right)^{0.5}$ where $k_{B}$ and $T$ are the Boltzmann constant and $T$ is the absolute temperature, respectively. ${ }^{6}$

\section{B. Biotinylation of $\lambda$-phage double-stranded DNA and coupling to streptavidin-coated beads}

Biochemical modification of the $\lambda$-DNA is required for force measurements to ensure a tethering process between the DNA and two $3180 \mathrm{~nm}$ streptavidin-coated beads. Therefore, $\lambda$-dsDNA (Promega Corp., WI) was heated up to $323 \mathrm{~K}$ for 5 min to convert the circular form of the DNA into the linearized form, yielding so called "sticky ends" containing 12 free bases at both $5 \mathrm{ft}$. ends of the $\lambda$-DNA. Biotin-14dCTP (GibcoBRL, Invitrogen Corp., CA) and dATP, dGTP, and dTTP (Amersham Biociences UK Ltd., UK) were added to both $3 \mathrm{ft}$. ends using Klenow-exo ${ }^{-}$polymerase enzyme (New England Biolabs Inc., MA). After intensive cleaning with Microcon-YM-50 cellulose filters (Amicon Microcon, Millipore Corp., MA) we ended up with modified dsDNA molecules with biotin groups at their $3 \mathrm{ft}$. ends. The biotinylated $\lambda$-DNA was stored as a dilution of $15 \mathrm{pM}$. DNA binding ligands (YOYO-1, ethidium bromide, and distamycin-A) are used as dilution of $1 \mu \mathrm{M}$, respectively.

The buffer was always filtered with syringe filters $(0.22$ $\mu \mathrm{m}$, Qualilab, Germany). Additionally the solution of $\lambda$-DNA and the solution with streptavidin-coated beads were degased to prevent spouting air bubbles inside the tubings or the sample chamber while performing an experiment. All experiments were carried out in $10 \mathrm{mM}$ Tris buffer $(p \mathrm{H} \mathrm{8.0)}$ containing $150 \mathrm{mM} \mathrm{NaCl}$ and the respective concentrations of the drug/dye. The concentration of the DNA in the sample chamber was $2 \mathrm{pM}$.

Briefly, the working procedure was as follows: After filling the cell with buffer, bead solution $\left(5 \times 10^{-4} \% \mathrm{w} / \mathrm{v}\right)$ was injected. Once a bead was trapped, it was transferred to the tip of the micropipette. A second bead stays trapped inside the laser focus. After rinsing the sample chamber with buffer, $\lambda$-DNA solution was injected and a flow of $800 \mu \mathrm{m} / \mathrm{s}$ inducing a drag force of about $24 \mathrm{pN}$ was established.

Once a DNA molecule attaches to the trapped bead, an increased force signal of about 2.5 to $3.0 \mathrm{pN}$ can be detected 

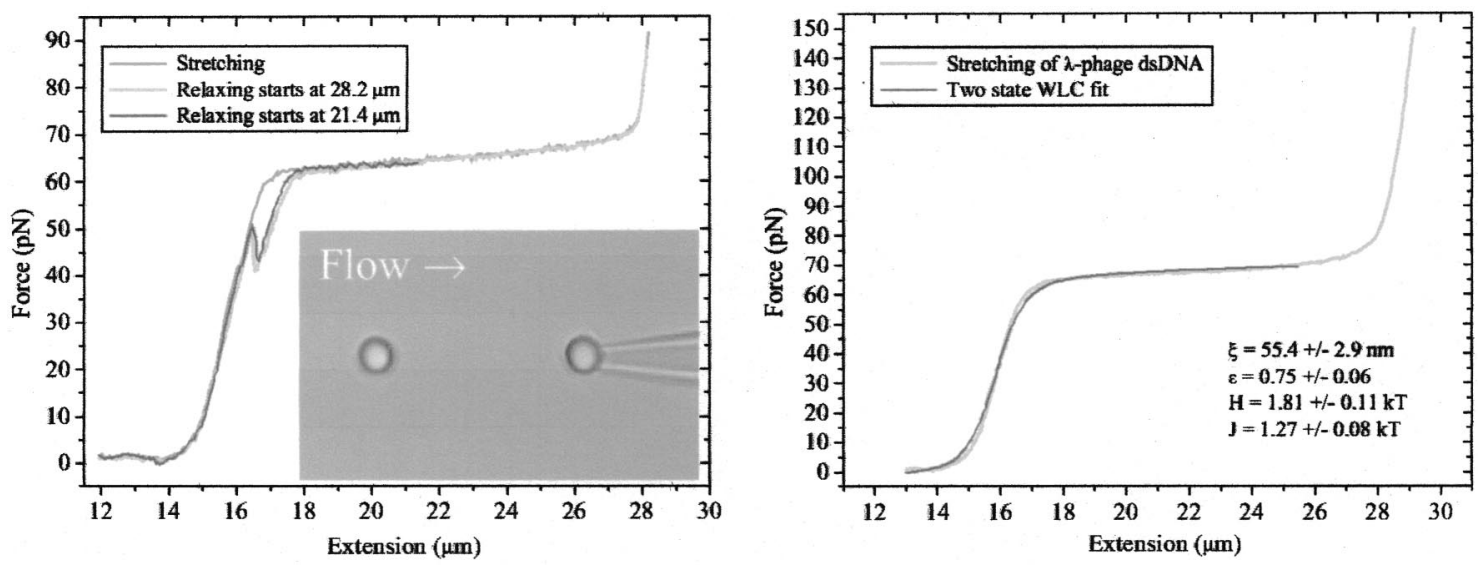

FIG. 4. (Left-hand side) Overstretching of $\lambda$-phage dsDNA in a force-extension/relaxation loop. Reproducible force hysteresis effects can be identified during relaxation. Inset: Single video frame obtained during the optical tweezers experiment. A bead (3.18 $\mu \mathrm{m}$ diameter) coated with streptavidin is held on a micropipette. A second bead (left-hand side) is trapped in the laser beam. A single biotinylated dsDNA molecule is attached to both streptavidin-coated spheres. (Right-hand side) two-state wormlike chain fit of the experimental data of free $\lambda$-phage dsDNA elasticity at the B-S transition and force-extension data of stretching dsDNA at higher laser power $(900 \mathrm{~mW})$ reveals high precision measurements and an extended force range up to $150 \mathrm{pN}$.

due to hydrodynamic drag forces of the elongated DNA in the buffer flow. The free end of the DNA was coupled to the bead fixed on the micropipette by reducing the distance to 11 $\mu \mathrm{m}$, which is smaller than the contour length of the $\lambda$-DNA molecule $(16.4 \mu \mathrm{m}) .{ }^{11}$ Tethering of the DNA between the two beads can be checked by a careful extension of the molecule where an attractive force signal indicates proper fixation.

\section{RESULTS AND DISCUSSION}

Figure 4 (left-hand side) shows three force versus extension/relaxation curves obtained from overstretching a single $\lambda$-DNA molecule. The experimental noise of the force experiment even at higher laser power $(950 \mathrm{~mW})$ is well below $1.5 \mathrm{pN}$ (Fig. 4, right-hand side) while achieving a maximal force of $150 \mathrm{pN}$. The noise level is dominated by fluctuations due to mechanical noise and of the flow $(<0.2$ $\mathrm{pN}$ ) and laser intensity fluctuations (less than $0.4 \mathrm{pN}$ in a frequency bandwith of 0.1 to $1 \mathrm{~Hz}$ at $600 \mathrm{~mW}$ ). Furthermore, thermal noise caused by Brownian motion has to be considered as well.

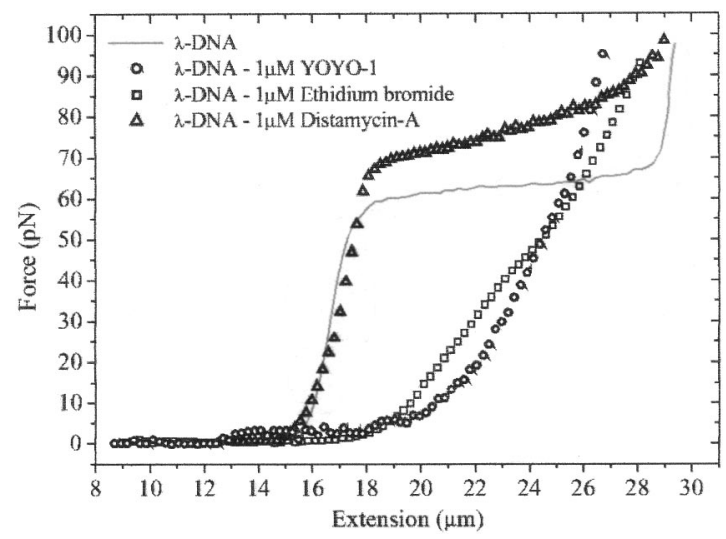

FIG. 5. Changes in the force response of $\lambda$-phage dsDNA in the presence of DNA binding agents. $\lambda$-phage dsDNA reference, YOYO- 1 intercalated $\lambda$-DNA, EtBr intercalated $\lambda$-DNA, and $\lambda$-DNA complexed with Distamycin-A.
The $B-S$ transition ${ }^{11}$ indicated by the plateau is reached at forces of about $65 \mathrm{pN}$. After the first overstretching (Fig. 4, left-hand side velocity: $30 \mathrm{~nm} / \mathrm{s}$ ); the DNA was relaxed at the same velocity showing a very high reproducibility, except for a small hysteretic variation at the onset of the transition from $B-S$ overstretching to $B$-form elasticity. This observation was found to be very reproducible as indicated in the second relaxation curve of Fig. 4 and interpreted as single-stranded nicks of the DNA. ${ }^{11}$

The force-extension curve in the $B-S$ transition regime was fitted using a "two-state wormlike chain" model $^{20}$ (Fig. 4 , right-hand side). The values for the persistence length $\xi$, the fractional elongation $\varepsilon$, the free energy difference per segment $2 H$ and the correlation energy between adjacent segments $J$ were determined to be $\xi=55.4 \pm 2.9 \mathrm{~nm}, \varepsilon$ $=0.75 \pm 0.06, H=1.81 \pm 0.11 k_{B} T$, and $J=1.27 \pm 0.08 k_{B} T$, respectively, and are in excellent agreement with the results of Ahsan et al. ${ }^{20}$

Effects of different DNA binding drugs on the overstretching behavior of DNA were investigated using this optical tweezers setup. In Fig. 5, four force-extension curves are presented, exhibiting the different force responses of $\lambda$-DNA to binding of a bis-intercalator (YOYO-1), ${ }^{14}$ an intercalator (ethidium bromide), ${ }^{12,15,17}$ and a minor groove binder acting as an antitumor drug (distamycin-A). ${ }^{14}$

We could unambiguously show that mechanical properties of DNA vary in the presence of different binding agents, which opens new possibilities to use this setup as a singlemolecule biosensor to investigate DNA binding agents and even to identify molecular binding mechanisms.

\section{ACKNOWLEDGMENTS}

Technical assistance from Christoph Pelargus and Gerd Wiebusch (Bielefeld University) is gratefully acknowledged. The authors thank Martin Hegner and Wilfried Grange (University of Basel, Switzerland) for providing technical advice, biochemical preparation protocols, and functionalized DNA molecules in the early stage of this work. 
${ }^{1}$ A. Ashkin, Phys. Rev. Lett. 24, 156 (1970).

${ }^{2}$ A. Ashkin, J. M. Dziedzic, J. E. Bjorkholm, and S. Chu, Opt. Lett. 11, 288 (1986).

${ }^{3}$ A. Ashkin, Phys. Rev. Lett. 40, 729 (1978).

${ }^{4}$ A. Ashkin, Biophys. J. 61, 569 (1992).

${ }^{5}$ A. Ashkin, Proc. Natl. Acad. Sci. U.S.A. 94, 4853 (1997).

${ }^{6}$ L. P. Ghislain, N. A. Switz, and W. W. Webb, Rev. Sci. Instrum. 65, 2762 (1994).

${ }^{7}$ A. Ashkin and J. M. Dziedzic, Science 235, 1517 (1987).

${ }^{8}$ K. Svoboda and S. M. Block, Annu. Rev. Biophys. Biomol. Struct. 23, 247 (1994).

${ }^{9}$ S. Yamada, D. Wirtz, and S. C. Kuo, Biophys. J. 78, 1736 (2000).

${ }^{10}$ S. M. Block, L. S. Goldstein, and B. J. Schnap, Nature (London) 348, 6299 (1990)

${ }^{11}$ S. B. Smith, Y. Cui, and C. Bustamante, Science 271, 795 (1996).
${ }^{12}$ D. Anselmetti, J. Fritz, B. Smith, and X. Fernandez-Busquets, Single Mol. 1, 17 (2000)

${ }^{13}$ R. Krautbauer, L. H. Pope, T. E. Schrader, S. Allen, and H. E. Gaub, FEBS Lett. 510, 154 (2002).

${ }^{14}$ R. Eckel, R. Ros, A. Ros, S. D. Wilking, N. Sewald, and D. Anselmetti, Biophys. J. 85, 1968 (2003).

${ }^{15}$ S. Husale, W. Grange, and M. Hegner, Single Mol. 3, 91 (2002).

${ }^{16}$ P. Cluzel, A. Lebrun, C. Heller, R. Lavery, J. L. Viovy, D. Chatenay, and F. Caron, Science 271, 792 (1996)

${ }^{17}$ E. Krautbauer, S. Fischerlander, S. Allen, and H. E. Gaub, Single Mol. 3, 97 (2002)

${ }^{18}$ C. Bustamante, J. C. Macosko, and G. J. Wuite, Nat. Rev. Mol. Cell. Biol. 1, 130 (2000)

${ }^{19}$ M. W. Allersma, F. Gittes, M. J. de Castro, R. J. Steward, and C. F. Schmidt, Biophys. J. 74, 1074 (1998).

${ }^{20}$ A. Ahsan, J. Rudnick, and R. Bruinsma, Biophys. J. 74, 132 (1998). 